\title{
INJECTION OF THE BRONCHIAL CIRCULATION IN A CASE OF TRANSPOSITION
}

\author{
BY \\ L. CUDKOWICZ* AND J. B. ARMSTRONG $\dagger$ \\ From the Department of Medicine, Postgraduate Medical School, London \\ Received January 10, 1952
}

Enlargement of the bronchial arteries in a case of pulmonary stenosis was first mentioned by Jacobson (1816). More recently East and Barnard (1938), Campbell and Gardner (1950), GrosseBrockhoff et al. (1950), and Allanby et al. (1950) reported increases in the bronchial circulation in pulmonary atresia. In the experience of Taussig (1947), hypertrophy of the bronchial arteries is frequently seen in pulmonary stenosis and atresia and in persistent truncus arteriosus.

The present case concerns the combination of hypertrophied bronchial arteries with a patent, transposed pulmonary trunk, a right sided aorta, and a ventricular septal defect.

\section{CASE REPORT}

The patient, a girl, aged 3, was admitted to the Children's Unit of Hammersmith Hospital under the care of Dr. Mann. She became blue on the sixth day after birth and squatted all her life. Her fingers and toes were clubbed and a soft systolic murmur, loudest at the tricuspid area, was conducted posteriorly and was audible along the medial border of the left scapula. This suggested enlargement of the bronchial arteries as emphasized by Campbell and Gardner (1950). The blood pressure was $100 / 60$ : the hæmoglobin, $25.6 \mathrm{~g}$. per $100 \mathrm{ml}$; red blood corpuscles 9.9 millions. The cardiogram showed right ventricular preponderance. On screening, the lung fields seemed oligæmic (Fig. 1) and the pulmonary arteries were difficult to identify.

A Blalock operation was performed by Mr. W. P. Cleland. The pulmonary artery pressure was thought to be reduced, and the left subclavian artery was inserted into the left pulmonary artery. On releasing the clamp from the pulmonary artery, the colour of the child changed to pink. Twenty hours later, sudden dyspnœa and cyanosis developed, and the child succumbed to pulmonary œdema.

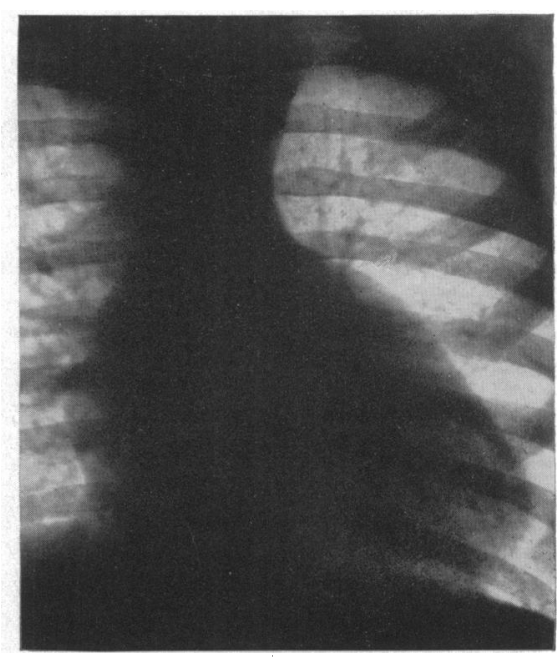

Fig. 1.-Teleradiogram of the heart and lung fields.

Post-mortem findings. The body of the child was undersized. The chest and abdomen were opened and the veins everywhere showed engorgement. The kidneys and liver were enlarged, but the spleen was of normal size. No fluid or adhesions were present in the pleural sacs and the lungs were œdematous. The heart lay in a horizontal position and was hypertrophied. The aorta arose from the anterior (right) ventricle, and two normal sized pulmonary arteries emerged from behind the aorta. The pulmonary trunk came from the posterior (left) ventricle. The left subclavian

* In receipt of a grant from the Medical Research Council. †Now at the Department of Medicine, University of Manitoba, Canada. 


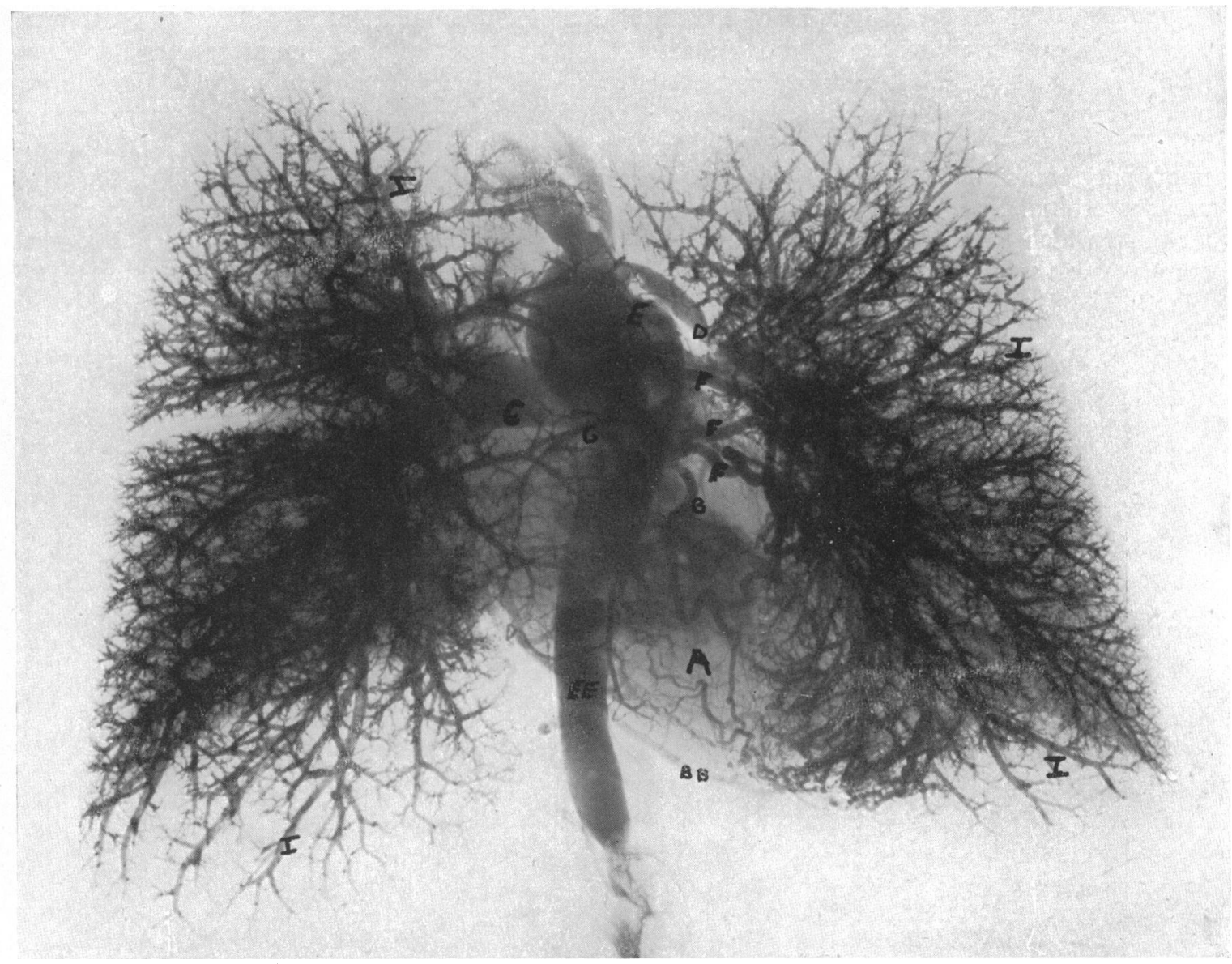

Fig. 2.- Radiograph of injected thoracic contents viewed in an antero-posterior position. (A) Heart. (B) Left and (BB) right coronary artery. (C) Right pulmonary artery. (D) Left subclavian anastomosis. (E) Ligature around descending thoracic aorta (EE) Descending aorta. (F) Left bronchial arteries. (G) Right bronchial artery. (I) Pulmonary artery bed. One-third of normal size.

anastomosis was secure and no leakage was evident. The total thoracic content was then removed in bulk.

Anatomical findings after injection. The descending aorta was firmly clamped beyond the origin of the left subclavian artery, and a cannula introduced into the distal end of the descending thoracic aorta. The technique of the injection of the bronchial arteries and the radio-opaque medium used were the same as described by Cudkowicz and Armstrong (1951). The medium does not penetrate vessels of less than $60 \mu$ in diameter.

Following the injection, the heart was opened. A minute hole between the atria could be demonstrated. A large ventricular septal defect was present in the middle third of the septum. Both ventricular muscles were hypertrophied, and one cusp of the pulmonary valve was deformed and thickened: this could have caused some obstruction to the outflow. The ductus arteriosus was not patent. The bronchial arteries were followed from the hila along the main bronchi of each lung, and evidence of direct communication between the trunks of the bronchial and pulmonary arteries could not be found. A network of very fine arterioles, filled with the injection medium, was noted to course across the adventitia of the pulmonary arteries. It was thought that these vessels were dilated vasa vasorum which are normally derived from the bronchial arteries (Miller, 1947). 
Radiography. The radiographs of the injected thoracic content, taken before the heart was opened, showed that three large bronchial arteries entered the left lung hilum after emerging from the lateral aspect of the descending aorta. A large branch derived from the uppermost of the three arteries crossed the aorta posteriorly and entered the right hilum. A fourth bronchial artery arising immediately below the other three coursed directly from the aorta to the right hilum. The intrapulmonary course of the vessels became lost because of the complete superimposition of the pulmonary arterial trees, which had filled indirectly through the bronchial arteries. The injection medium, which had traversed intrapulmonary anastomoses to reach the pulmonary arteries, was conveyed to the ascending aorta and its branches through the patent subclavian anastomosis (see Fig. 2).

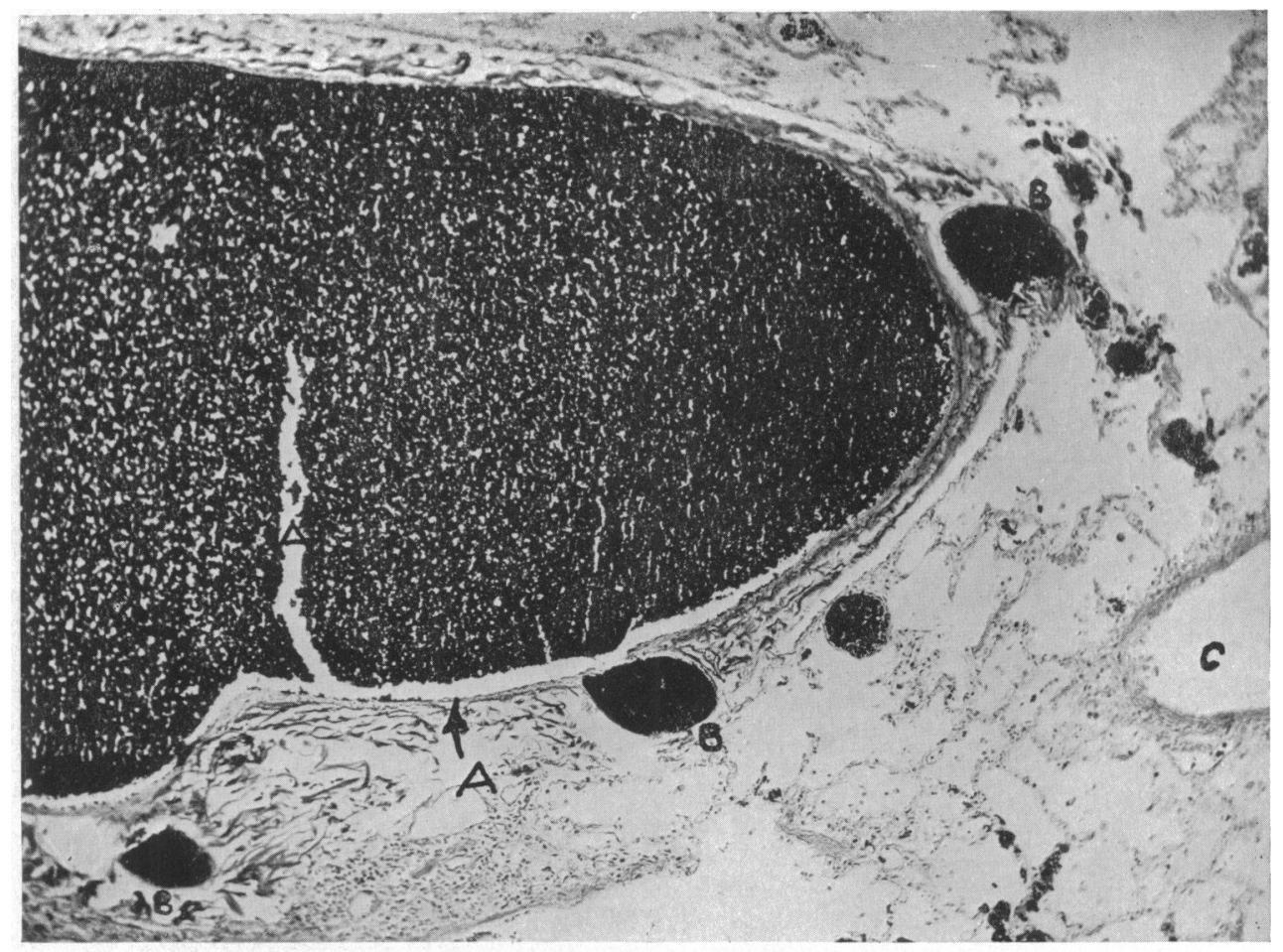

FIG. 3.-Photomicrograph of an intrapulmonary branch of a pulmonary artery approximately two inches from the left hilum. (A) Dilated pulmonary artery filled with bismuth granules. (B) Densely filled vasa vasorum in the adventitia. (C) Empty pulmonary vein. (Magnification, $\times 75$ )

Histology and serial sections. The injection medium fixed well and allowed the injected vessels to be traced histologically. The medium appeared as a mass of black granules in vessels of more than $60 \mu$ in diameter.

Cuts were examined along broncho-vascular bundles from the hila to the periphery. These showed no alteration in the pattern of the bronchial arteries to the various intrapulmonary structures which they normally supply. The walls of the bronchi were unchanged, and normal-sized bronchial arterioles were visible in the mucosa, substantia propria, and near the cartilages. The pulmonary arteries accompanying these bronchi were also well injected, but less densely. The walls of the pulmonary arteries appeared thinner than normal and many dilated vasa vasorum in the adventitia communicated freely with their lumina (see Fig. 3 and 4). Sections from the lung periphery showed filling of all intrapulmonary arterioles, but those normally derived from the bronchial arteries, 
such as interlobular septal or pleural arterioles, were more densely filled. The pulmonary veins and capillaries remained uninjected throughout, because of the inability of the injection medium to penetrate vessels less than $60 \mu$ in diameter.

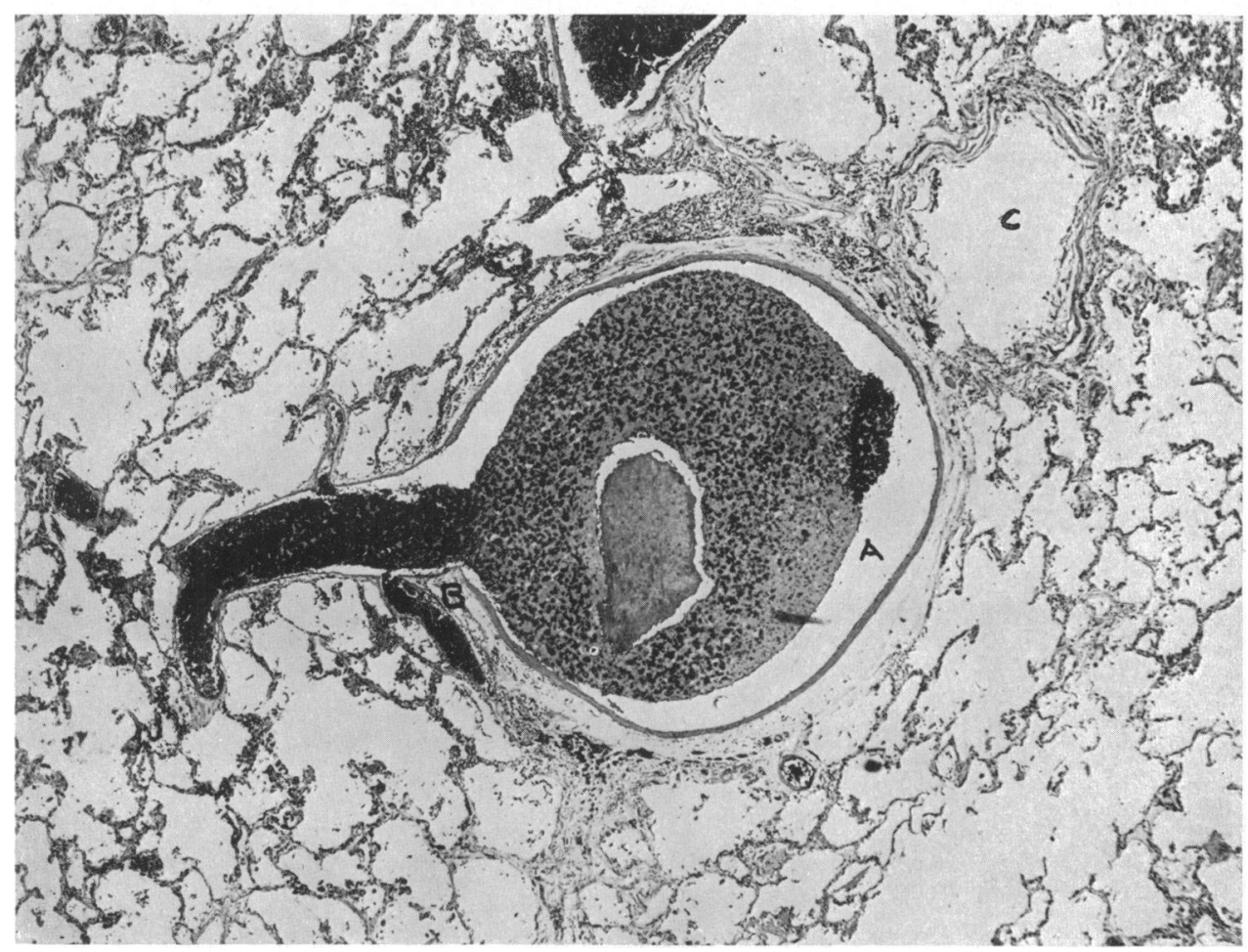

Fig. 4.-Photomicrograph of pulmonary arteriole in the lung periphery. (A) Dilated and injected pulmonary arteriole. (B) Dilated communicating and densely injected vas. (C) Accompanying small bronchi. (Magnification, $\times 35$.)

\section{Discussion}

The post-mortem radiographical and histological study of the injected bronchial circulation in this case suggests that bronchial artery hypertrophy occurred in the presence of patent pulmonary arteries, although some reduction in total pulmonary artery blood flow may have been caused by the deformed cusp of the pulmonary valve. A considerable volume of deoxygenated blood reached the pulmonary capillaries from the right sided aorta through the bronchial arteries, and this was conveyed to the pulmonary arteries as a result of the enlargement and direct anastomoses of the vasa vasorum with the pulmonary artery lumina. No evidence of direct shunts between the trunks of the bronchial and pulmonary arteries could be seen, and the distribution of the bronchial arteries within those intrapulmonary structures that they normally supply was unaltered. The pre-capillary anastomoses between the two circulations appear, therefore, to have been brought about by the dilatation and penetration of the vasa vasorum which are normally present in the adventitia and media of the pulmonary arteries. Augmented bronchial blood flow has been thought to follow obstruction to the pulmonary arteries, (Virchow, 1856; Taussig, 1947). Karsner and Ash (1912) believed that diminution in pulmonary artery pressure alone could also increase the flow through the bronchial arteries: the mechanism of the readjustment is not known. In histological studies of injected bronchial arteries in tuberculosis, certain types of pulmonary emphysema, lung neoplasms, and bronchiectasis where thrombosis of the pulmonary arteries was apparent, recanalization of the occluded lumina by vigorously proliferating vasa vasorum was demonstrable (Cudkowicz, 1951). It would appear, therefore, that these small branches of the bronchial arteries, which do not normally communicate directly with the lumina of the pulmonary arteries (Miller, 1947), can in the presence of diminished pulmonary artery blood flow or pul- 
monary artery occlusion effect pre-capillary anastomoses, which in turn become associated with hypertrophy of the bronchial arteries. The presence of enlarged bronchial arteries in congenital heart diseases associated with patent pulmonary arteries suggest that the pulmonary vascular capacity is already utilized to its maximum, and this may very well constitute a contra-indication for further operative augmentation of pulmonary blood flow.

\section{SUMMARY}

The bronchial circulation was studied at necropsy in a case of transposition of the great vessels with ventricular septal defect and closed ductus arteriosus. Enlargement of the bronchial arteries and direct wide-spread pre-capillary anastomoses by dilated vasa vasorum with the pulmonary arteries was demonstrated. The significance of these findings in regard to further surgical augmentation of pulmonary blood flow is discussed.

We desire to thank Professor J. McMichael and Dr. C. V. Harrison for the provision of facilities, Dr. Travor Mann and Mr W. P. Cleland and Dr. Duncan-White for permission to consult the clinical findings and radiographs, the technical staff of the Departments of Medicine and Pathology of the Postgraduate Medical School, London, for their help, Mr. E. V. Willmott, F.R.P.S. for the photomicrography, and Mr. K. Moreman, A.R.P.S. for reproducing the radiograph.

\section{REFERENCES}

Allanby, J. D., Brinton, W. D., Campbell, M., and Gardner, F. (1950). Guy's Hosp. Rep., 99, 2, 110.

Campbell, M., Gardner, F. (1950). Brit. Heart J., 12, 202.

Cudkowicz, L. (1951). M.D. Thesis., 1951. University of London.

, and Armstrong, J. B., (1951). Thorax, 6, 343.

East, T., and Barnard, W. G. (1938). Lancet, 1, 834.

Grosse-Brockhoff, F., Neuhaus, G., and Schaede, A. (1950). Deut. Arch. klin. Med., 197, 621.

Jacobsen, - . (1816). Deut. Arch. Physiologie, (Heckel), 2, 134.

Karsner, H. K., and Ash, H. E. (1912). J. exp. Med., 27, 205.

Miller, W. S. (1947). The Lung. 2nd ed., Springfield, Ill., C. Thomas.

Taussig, H. B. (1947). Congenital Malformations of the Heart The Commonwealth Fund. New York, p. 464.

Virchow, R. (1856). Gesammelte Abhandlungen wissenschaflicher Medizin., 20, 285. 